EDITORIAL

\title{
Standardisation of protocols can be crucial in long non-coding RNA research
}

(c) The Author(s), under exclusive licence to Springer Nature Limited 2021

In this issue, Traversa et al. [1] reviewed our current knowledge about the role of circular and linear forms of PVT1 non-coding RNA in cancer and human diseases. They highlighted the technical challenges of these studies and raised a potential bias in the publications, which require more attention from researchers.

British Journal of Cancer (2022) 126:833-834; https://doi.org/ $10.1038 / \mathrm{s} 41416-021-01656-8$

\section{MAIN}

Along with the increasing number of discovered circular RNAs (circRNAs) the need of understanding their functional roles became an important topic. Plasmacytoma variant translocation 1 (PVT1) IncRNA gene produces both circular (circPVT1) and linear (IncPVT1) transcripts, and their expression has been found to correlate with tumour progression in different types of cancers.

Traversa et al. reviewed the literature about the IncPVT1 and circPVT1 transcripts, which have been reported to have similar functions in the pathophysiology of diseases such as solid tumours and haematological malignancies. They highlighted in Table 2 the frequent association between IncPVT1 and circPVT1 upregulation and poor prognosis of the patients with different types of cancers, including but not limited to colorectal, ovarian and gastric cancer [1]. Therefore, both the linear and the circular transcripts have been implicated to be potential prognostic biomarkers.

The authors suggest, that since the two molecules share the IncPVT1 exon 2 sequence, it is difficult to distinguish the expression of the two transcripts, which can result in a biased interpretation of the data. They pointed out that some of the observations regarding the shared functions of these transcripts can be a result of this technical issue, and therefore lead to incorrect conclusions.

Even though their review focus on the expression of circular and linear PVT1, their point goes beyond that. Their case raises attention to the potentially misleading interpretation of data during the investigation of circRNAs and can be valid for other long non-coding RNAs as well.

This review highlights the importance of solid understanding and the appropriate choice of the technical approaches to be used for the dissection of complex genomic loci. Besides, using several independent techniques to underneath experimental findings can be also critical.

The issue raised in the review is not entirely new. Researchers have been discussing about a "replication crisis" in science, including cancer research, for several years now, referring to the uncovered phenomena that a lot of published data cannot be reproduced due to different reasons. This reminds us that despite the constant publication pressure and the difficulties of publish negative results, a thoroughly understanding of the used methods, careful planning of the experiments, preliminary measurements and application of different independent methods are extremely important. Besides, this can lead to expanding the discussion in the scientific community about standardise the experimental protocols and the interpretation of the data.

Generating and following guidelines similar to the MIQE (Minimum Information for Publication of Quantitative Real-Time PCR Experiments) guideline can highly increase the reproducibility and reliability of the results.

\section{OUTLOOK}

During these days, when generating a large amount of data is easily accessible, it is especially important to generate clear criteria on how we can improve our daily routine to not only generate a huge amount of data but to be sure that those data are going to be reliable, durable, and be a valuable part of our common scientific knowledge. Moreover, it is important to give space for debate and be open for discussion about how we can improve our standards.

Kinga Németh ${ }^{1}$ and George A. Calin (iD ${ }^{1,2}$ 压 ${ }^{1}$ Department of Translational Molecular Pathology, The University of Texas, MD Anderson Cancer Center, Houston, TX, USA. ${ }^{2}$ Center for RNA Interference and Non-coding RNAs, The University of Texas MD Anderson Cancer Center, Houston, TX, USA.

凶email: gcalin@mdanderson.org

\section{REFERENCE}

1. Traversa D, Simonetti G, Tolomeo D, Visci G, Macchia G, Ghetti M, et al. Unraveling similarities and differences in the role of circular and linear PVT1 in cancer and human disease. Br J Cancer. 2021 (this issue).

\section{AUTHOR CONTRIBUTIONS}

$\mathrm{KN}$ and GAC equally contributed to the manuscript writing.

\section{FUNDING}

The authors received no specific funding for this work.

\section{ETHICS APPROVAL AND CONSENT TO PARTICIPATE}

No biological samples or patient data were used for this work.

Received: 4 November 2021 Revised: 13 November 2021 Accepted: 23 November 2021

Published online: 15 December 2021 
834

CONSENT TO PUBLISH

Not applicable.

\section{COMPETING INTERESTS}

GAC is the scientific founder of Ithax Pharmaceuticals. KN declares no competing interests.

\section{ADDITIONAL INFORMATION}

Correspondence and requests for materials should be addressed to George A. Calin.

Reprints and permission information is available at http://www.nature.com/ reprints

Publisher's note Springer Nature remains neutral with regard to jurisdictional claims in published maps and institutional affiliations. 